\title{
High preoperative neutrophil-lymphocyte ratio predicts poor survival in patients with gastric cancer
}

\author{
Hideaki Shimada, Nobuhiro Takiguchi, Osamu Kainuma, Hiroaki Soda, Atsushi Ikeda, Akihiro Cho, \\ Akinari Miyazaki, Hisashi Gunji, Hiroshi Yamamoto, and Matsuo Nagata \\ Department of Gastroenterological Surgery, Chiba Cancer Center, Chiba, Japan
}

\begin{abstract}
Background. The neutrophil-lymphocyte ratio (NLR) reflects inflammatory status. An elevated NLR has been reported to be a prognostic indicator in some malignant tumors. The aim of this study was to evaluate the clinical significance of the preoperative NLR in patients with primary gastric cancer. Methods. A total of 709 men and 319 women, with a mean age of 64.4 years, who underwent gastrectomy were included. The numbers of patients in each pathological stage were as follows: stage I, 584; stage II, 132; stage III, 153; and stage IV, 159. The mean NLR was $2.62 \pm 1.68$. A total of 127 patients $(12.4 \%)$ with an NLR of 4.0 or more were classified as high NLR individuals in this study. The prognostic significance of a high NLR, together with various clinicopathological factors, was evaluated by multivariate analysis.

Results. The 5-year survival of patients with a high NLR was significantly worse than that of patients with a low NLR $(57 \%$ vs $82 \%, P<0.001)$. Univariate and multivariate analyses of clinicopathological factors affecting survival revealed that high NLR, depth of tumor, positive lymph nodes, distant metastasis, peritoneal metastasis, poorly differentiated type, and high platelet count were significant risk factors for reduced survival. On multivariate analysis, after adjusting for tumor stage, a high NLR was an independent risk factor for reduced survival $(P=0.003$; adjusted hazard ratio, 1.845 ; $95 \%$ confidence interval, 1.236-2.747).

Conclusion. A high preoperative NLR may be a convenient biomarker to identify patients with a poor prognosis after resection for primary gastric cancer.
\end{abstract}

Key words Neutrophil-lymphocyte ratio - Gastric cancer · Survival

Offprint requests to: $\mathrm{H}$. Shimada

Department of Surgery, Toho University School of Medicine, 6-11-1 Omori-Nishi, Ota-ku, Tokyo 143-8541, Japan

Received: September 15, 2009 / Accepted: March 15, 2010

\section{Introduction}

Although preoperative tumor staging is useful to select the appropriate therapeutic strategy for patients with gastric cancer, clinical tumor staging alone cannot predict patients' prognosis. A few serum markers were found to be associated with poor prognosis in patients with gastric cancer and are therefore useful for monitoring and predicting early recurrence and poor prognosis $[1,2]$. The host inflammatory response to cancer cells is also associated with tumor progression $[3,4]$. In advanced tumors, a high preoperative C-reactive protein level and/or high platelet count were frequently observed and were associated with poor patient prognosis $[5,6]$. The preoperative neutrophil-lymphocyte ratio (NLR) also reflects patients' inflammation status, clinical stage, and patients' survival in colon cancer, lung cancer, and liver cancer [7-12]. Increased numbers of neutrophils and/or decreased numbers of lymphocytes may suppress lymphokine-activated killer cells, thereby increasing the propensity to metastasis [13]. However, only limited information on the clinical significance and prognostic significance of NLR in patients with gastric cancer has been reported [14-17]. In this study, we measured the preoperative NLR in 1028 patients with primary gastric cancer to determine its clinicopathological and prognostic significance.

\section{Patients and methods}

\section{Patients}

Between 2001 and 2007, a total of 1028 patients with primary gastric adenocarcinoma who underwent gastrectomy at the Department of Gastroenterological Surgery of Chiba Cancer Center were investigated. The patient population consisted of 709 men $(69 \%)$ and 319 women $(31 \%)$, with a median age of 65 years (range, 26 
to 89 years). All patients underwent either a total or subtotal gastrectomy with standard lymphadenectomy. The resected specimens were pathologically classified according to the $13^{\text {th }}$ edition ( $2^{\text {nd }}$ English edition) of the Japanese classification of gastric carcinoma [18]. The distribution of cancer stages was as follows: stage I, 584; stage II, 132; stage III, 153; and stage IV, 159. All patients were followed up regularly until December 2008 or until death. The follow-up period ranged from 12 months to 84 months (median, 23 months).

\section{Blood sample analyses}

Peripheral blood was obtained at the time of diagnosis before surgery. The numbers of white blood cells and platelets were determined with a hemocytometer. The percentages of particular types of cells were determined by using a Coulter LH 750 Hematology Analyzer (Coulter, Brea, CA, USA). Absolute counts of particular cells were calculated by multiplying the percentage of particular cells by the number of white blood cells. NLR values were expressed as means \pm SD. An NLR value of 4.0 was used as a cutoff value,. classify high NLR $(\geq 4)$ and low NLR groups.

\section{Statistical analyses}

NLR values were compared using the Mann-Whitney $U$-test. Fisher's exact probability test was used to determine the significance of differences between two groups. Survival probabilities were calculated using the product limit method of Kaplan and Meier, considering overall deaths. Survival differences between groups were determined using the log-rank test. The influence of each significant predictor identified by univariate analysis was assessed by multivariate analysis using Cox's proportional hazards model. The influence of each clinicopathological variable on the risk of high NLR was assessed by logistic regression analysis. All statistical analyses were carried out using Stat View 5.0 for Windows (SAS Institute, Cary, NC, USA). All $P$ values less than 0.05 were considered statistically significant.

\section{Results}

\section{Preoperative NLR and clinicopathological variables}

The mean preoperative NLR in the 1028 patients was $2.62 \pm 1.68$, with a median of 2.23 (range, 0.38 to 21.86 ). The distribution of all patients according to NLR was as follows; NLR $<1.0, n=32 ; 1.0 \leq \mathrm{NLR}<2.0, n=381$; $2.0 \leq \mathrm{NLR}<3.0, n=321 ; 3.0 \leq \mathrm{NLR}<4.0 ; n=167 ; 4.0$ $\leq \mathrm{NLR}<5.0, n=75$; and $5.0 \leq \mathrm{NLR}, n=52$. We then analyzed the data to determine statistically significant differences between each combination of groups according to NLR. Log-rank $P$ values were calculated as $P=$ $0.102(\mathrm{NLR}<1.0$ vs $1.0 \leq \mathrm{NLR}<2.0), P=0.272(1.0<$ $\mathrm{NLR}<2.0$ vs $2.0 \leq \mathrm{NLR}<3.0), P=0.050(2.0 \leq \mathrm{NLR}<$ 3.0 vs $3.0 \leq \mathrm{NLR}<4.0), P=0.027(3.0 \leq \mathrm{NLR}<4.0$ vs $4.0 \leq \mathrm{NLR}<5.0)$, and $P=0.602$ (4.0 $\leq \mathrm{NLR}<5.0$ vs 5.0 $\leq \mathrm{NLR}$ ). Based on these results, we decided to use 4.0 as a cutoff value to predict patients' prognoses.

Based on the cutoff value of 4.0, 127 of the 1028 (12.4\%) patients were classified in the high NLR group. Significant differences were observed in the values of NLR according to age, tumor depth, nodal status, distant metastasis, peritoneal metastasis, and platelet count (Table 1). Significant differences were observed in the percentages of patients with high NLR according to tumor depth, nodal status, peritoneal metastasis, and platelet count (Table 1). Patients with peritoneal metastasis (32.7\%) most frequently had high NLR values.

NLR gradually increased with increasing tumor stage, from $2.37 \pm 1.41$ in stage I, $2.38 \pm 1.17$ in stage II, and $3.04 \pm 2.03$ in stage III to $3.34 \pm 2.25$ in stage IV (Fig. 1A). Although NLR values significantly increased according to tumor depth (Fig. 1B), NLR values did not significantly increase according to the extent of nodal metastases, N1, N2, and N3 (Fig. 1C). The percentage of patients with high NLR increased slightly in each stage, as follows; $8.4 \%$ in stage I, $9.8 \%$ in stage II, $19.6 \%$ in stage III, and $22.0 \%$ in stage IV (Fig. 1D).

To evaluate the effect of clinicopathological variables on NLR increases, logistic regression analysis was performed. Being elderly (age 65 or more) and/or being thrombocytotic were identified as independent risk factors for high NLR (Table 2).

\section{Prognostic significance of preoperative NLR}

A total of 147 patients died by the end of 2008; 128 of these 147 patients $(87 \%)$ died due to gastric cancer. To investigate whether a high NLR value reflected a poor prognosis for patients, a Kaplan-Meier analysis and a log-rank test were carried out. The overall survival rates gradually declined with increasing NLR. Based on the survival curves obtained after dividing the patients into six groups (Fig. 2A), 4.0 seemed to be an appropriate cutoff value to distinguish a high-risk group with a poor prognosis (Fig. 2B).

In regard to the clinicopathological factors evaluated, a significantly poor survival was observed in patients classified as elderly (age 65 or more), patients with T3 or T4, N1, M1, P1; poorly differentiated type; high platelet count; and high NLR (Table 3 ). After adjusting for TNM stages, high NLR groups showed significantly 
Table 1. Neutrophil-lymphocyte ratio according to clinicopathological variables

\begin{tabular}{|c|c|c|c|c|c|c|c|}
\hline Variables & & $\begin{array}{c}\text { Number of } \\
\text { patients }\end{array}$ & NLR & $P^{\mathrm{c}}$ & $\begin{array}{l}\text { Number of patients } \\
\text { with high } \text { NLR }^{\mathrm{d}}\end{array}$ & $(\%)$ & $P^{\mathrm{e}}$ \\
\hline \multirow[t]{2}{*}{ Sex } & Female & 319 & $2.54 \pm 1.37$ & 0.275 & 37 & 11.6 & 0.682 \\
\hline & Male & 709 & $2.66 \pm 1.80$ & & 90 & 12.7 & \\
\hline \multirow[t]{2}{*}{ Age (years) } & $<65$ & 489 & $2.48 \pm 1.32$ & 0.011 & 50 & 10.2 & 0.057 \\
\hline & $\geq 65$ & 539 & $2.75 \pm 1.95$ & & 77 & 14.3 & \\
\hline \multirow[t]{2}{*}{ Tumor depth } & $\mathrm{T} 1 \mathrm{~T} 2$ & 779 & $2.45 \pm 1.41$ & $<0.001$ & 77 & 9.9 & $<0.001$ \\
\hline & T3T4 & 249 & $3.16 \pm 2.25$ & & 50 & 20.1 & \\
\hline \multirow[t]{2}{*}{$\mathrm{N}$ factor } & No & 575 & $2.40 \pm 1.44$ & $<0.001$ & 51 & 8.9 & $<0.001$ \\
\hline & N1N2N3 & 453 & $2.91 \pm 1.91$ & & 76 & 16.8 & \\
\hline \multirow[t]{2}{*}{ Distant metastasis $^{\mathrm{a}}$} & M0 & 1001 & $2.58 \pm 1.63$ & $<0.001$ & 124 & 12.4 & $>0.999$ \\
\hline & M1 & 27 & $3.71 \pm 2.34$ & & 3 & 11.1 & \\
\hline \multirow[t]{2}{*}{ Peritoneal metastasis ${ }^{\mathrm{b}}$} & $\mathrm{P} 0$ & 976 & $2.52 \pm 1.52$ & $<0.001$ & 110 & 11.3 & $<0.001$ \\
\hline & $\mathrm{P} 1$ & 52 & $3.42 \pm 2.49$ & & 17 & 32.7 & \\
\hline \multirow[t]{2}{*}{ Histology } & Differentiated & 521 & $2.64 \pm 1.81$ & 0.796 & 65 & 12.5 & 0.925 \\
\hline & Poorly differentiated & 507 & $2.61 \pm 1.54$ & & 62 & 12.2 & \\
\hline \multirow[t]{2}{*}{ Platelet count } & $350 \times 10^{9} / 1>$ & 876 & $2.49 \pm 1.53$ & $<0.001$ & 81 & 9.2 & $<0.001$ \\
\hline & $350 \times 10^{9} / 1 \leq$ & 152 & $3.40 \pm 2.24$ & & 46 & 30.3 & \\
\hline
\end{tabular}

${ }^{a}$ Distant metastases include distant lymph node metastases and liver metastases

${ }^{\mathrm{b}}$ Peritoneal metastases include macroscopic peritoneal metastases and positive cytology

${ }^{\mathrm{c}} P$ values were calculated by Mann-Whitney $U$-test

${ }^{\mathrm{d}}$ Number of patients who showed high NLR, 4.0 or more

${ }^{\mathrm{e}} P$ values were calculated by Fisher's exact probability test

NLR, neutrophil-lymphocyte ratio

(A)

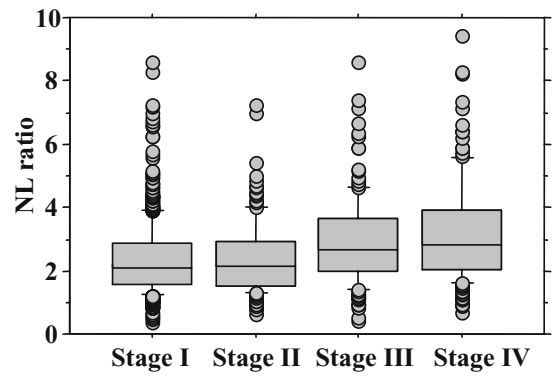

(B)

(C)

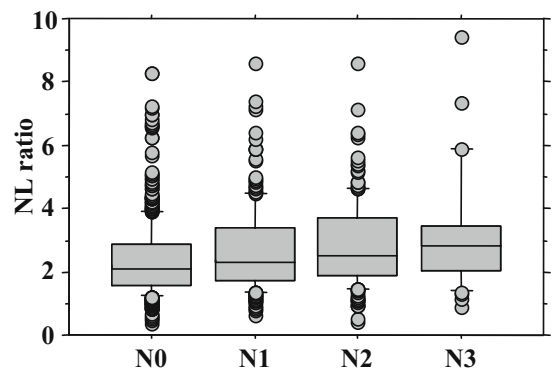

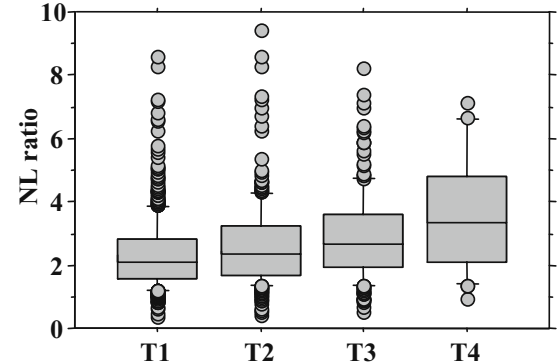

Fig. 1A-C. Neutrophil-lymphocyte $(N L)$ ratio according to pathological stage, tumor depth, and nodal status. A Stage (stage I vs II, $P<0.001$; stage II vs III, $P$ $=0.001 ;$ stage III vs IV; $P=0.217)$. B Tumor depth (T1 vs T2, $P<0.001$; T2 vs $\mathrm{T} 3, P=0.033 ; \mathrm{T} 3$ vs T4, $P=0.247)$. C Nodal status (N0 vs N1, $P=0.005 ; \mathrm{N} 1$ vs N2, $P=$ 0.169 ; N2 vs N3, $P=0.481)$. Horizontal lines within boxes represent median values, and boxes denote values between the 25 th and 75 th percentiles. Short horizontal lines indicate the 10th and 90th percentiles, respectively, and dots denote values outside these range limits. The $P$ values were determined using the MannWhitney $U$-test poorer survival than did low NLR groups in stages I, II, and IV, and a borderline difference was observed in stage III (Fig. 3). All significant prognostic factors and platelet counts tested by univariate analysis were evaluated by Cox's proportional hazards model. Tumor progression, high platelet count, poorly differentiated type, and high NLR were independent risk factors for reduced patient survival (Table 3 ).

\section{Discussion}

In the present study, a high preoperative NLR value in patients with gastric cancer was found to be associated with tumor progression and poor prognosis. The gradual increase in NLR may be caused by peritumoral inflammation produced by a large number of cancer cells in patients with advanced carcinoma. 
Table 2. Multivariate analysis of risk factors related to neutrophil-lymphocyte ratio in patients with gastric cancer

\begin{tabular}{|c|c|c|c|c|}
\hline Variables & & $\begin{array}{c}\text { Multivariate } \\
P^{\mathrm{c}}\end{array}$ & $\begin{array}{c}\text { Adjusted hazard } \\
\text { ratio }\end{array}$ & $\begin{array}{c}\text { Adjusted } 95 \% \\
\text { confidence interval }\end{array}$ \\
\hline \multirow[t]{2}{*}{ Sex } & Female & & & \\
\hline & Male & 0.910 & 0.976 & $0.636-1.496$ \\
\hline \multirow[t]{2}{*}{ Age (years) } & $<65$ & & & \\
\hline & $\geq 65$ & 0.045 & 1.503 & $1.010-2.237$ \\
\hline \multirow[t]{2}{*}{ Tumor depth } & $\mathrm{T} 1 \mathrm{~T} 2$ & & & \\
\hline & T3T4 & 0.219 & 1.399 & $0.818-2.393$ \\
\hline \multirow[t]{2}{*}{$\mathrm{N}$ factor } & No & & & \\
\hline & $\mathrm{N} 1 \mathrm{~N} 2 \mathrm{~N} 3$ & 0.287 & 1.286 & $0.809-2.045$ \\
\hline \multirow[t]{2}{*}{ Distant metastasis $^{\mathrm{a}}$} & M0 & & & \\
\hline & M1 & 0.135 & 1.762 & $0.838-3.708$ \\
\hline \multirow[t]{2}{*}{ Peritoneal metastasis $^{\mathrm{b}}$} & $\mathrm{P} 0$ & & & \\
\hline & $\mathrm{P} 1$ & 0.163 & 1.522 & $0.843-2.747$ \\
\hline \multirow[t]{2}{*}{ Histology } & Differentiated & & & \\
\hline & Poorly differentiated & 0.464 & 0.857 & $0.566-1.296$ \\
\hline \multirow[t]{2}{*}{ Platelet count } & $350 \times 10^{9} / 1>$ & & & \\
\hline & $350 \times 10^{9} / 1 \leq$ & $<0.001$ & 2.442 & $1.546-3.855$ \\
\hline
\end{tabular}

${ }^{a}$ Distant metastases include distant lymph node metastases and liver metastases

${ }^{\mathrm{b}}$ Peritoneal metastases include macroscopic peritoneal metastases and positive cytology

${ }^{\mathrm{c}}$ Logistic regression analysis

Table 3. Univariate and multivariate analyses of risk factors affecting survival in patients with gastric cancer

\begin{tabular}{|c|c|c|c|c|c|c|}
\hline Variables & & $\begin{array}{l}\text { 5-Year survival } \\
\text { rate }(\%)\end{array}$ & $\begin{array}{l}\text { Univariate } \\
\qquad P^{\mathrm{c}}\end{array}$ & $\begin{array}{l}\text { Multivariate } \\
\qquad P^{\mathrm{d}}\end{array}$ & $\begin{array}{c}\text { Adjusted } \\
\text { hazard ratio }\end{array}$ & $\begin{array}{c}\text { Adjusted } 95 \% \\
\text { confidence interval }\end{array}$ \\
\hline \multirow[t]{2}{*}{ Sex } & Female & 82 & & & & \\
\hline & Male & 76 & 0.221 & 0.209 & 1.266 & $0.876-1.832$ \\
\hline \multirow[t]{2}{*}{ Age (years) } & $<65$ & 83 & & & & \\
\hline & $\geq 65$ & 74 & 0.017 & 0.103 & 1.332 & $0.943-1.876$ \\
\hline \multirow[t]{2}{*}{ Tumor depth } & $\mathrm{T} 1 \mathrm{~T} 2$ & 91 & & & & \\
\hline & T3T4 & 38 & $<0.001$ & $<0.001$ & 2.604 & $1.046-2.257$ \\
\hline \multirow[t]{2}{*}{$\mathrm{N}$ factor } & N0 & 92 & & & & \\
\hline & N1N2N3 & 56 & $<0.001$ & $<0.001$ & 4.505 & $2.717-7.407$ \\
\hline \multirow[t]{2}{*}{ Distant metastasis $^{\mathrm{a}}$} & M0 & 82 & & & & \\
\hline & M1 & 0 & $<0.001$ & $<0.001$ & 4.831 & $1.215-2.717$ \\
\hline \multirow[t]{2}{*}{ Peritoneal metastasis ${ }^{\mathrm{b}}$} & $\mathrm{P} 0$ & 83 & & & & \\
\hline & $\mathrm{P} 1$ & 15 & $<0.001$ & 0.004 & 1.818 & $3.003-7.752$ \\
\hline \multirow[t]{2}{*}{ Histology } & Differentiated & 81 & & & & \\
\hline & Poorly differentiated & 72 & $<0.001$ & 0.018 & 1.548 & $1.079-2.222$ \\
\hline \multirow[t]{2}{*}{ Platelet count } & $350 \times 10^{9} / 1>$ & 81 & & & & \\
\hline & $350 \times 10^{9} / 1 \leq$ & 60 & $<0.001$ & 0.029 & 1.536 & $1.695-2.000$ \\
\hline \multirow[t]{2}{*}{ NLR } & $<4.0$ & 81 & & & & \\
\hline & $\geq 4.0$ & 56 & $<0.001$ & 0.003 & 1.845 & $1.236-2.747$ \\
\hline
\end{tabular}

${ }^{a}$ Distant metastases include distant lymph node metastases and liver metastases

${ }^{\mathrm{b}}$ Peritoneal metastases include macroscopic peritoneal metastases and positive cytology

${ }^{\mathrm{c}}$ Log-rank test

${ }^{\mathrm{d}}$ Cox proportional hazards model

NLR, neutrophil-lymphocyte ratio

Several studies have shown that high preoperative NLR values in patients with different types of carcinoma were associated with a poor prognosis [8-12]. Similarly, in our study, the prognosis of patients with a high NLR was significantly worse than that of patients with a low NLR. Because NLR was significantly associated with tumor progression, the difference in survival may be partly explained by differences in tumor volumes between the low and high NLR groups. After control- ling for these pathological parameters by multivariate analysis, the NLR was still shown to be an independent prognostic factor separate from TNM factors.

The cutoff value used in the present study was different from those in previous reports $(2.0,2.5$, or 5.0) [12, $14,15]$. We, first of all, analyzed overall survival curves according to each NLR value, from a minimum level $(<1.0)$ to the highest level $(5.0 \leq)$. Based on univariate analysis of these survival curves, 4.0 was the best cutoff 
(A)

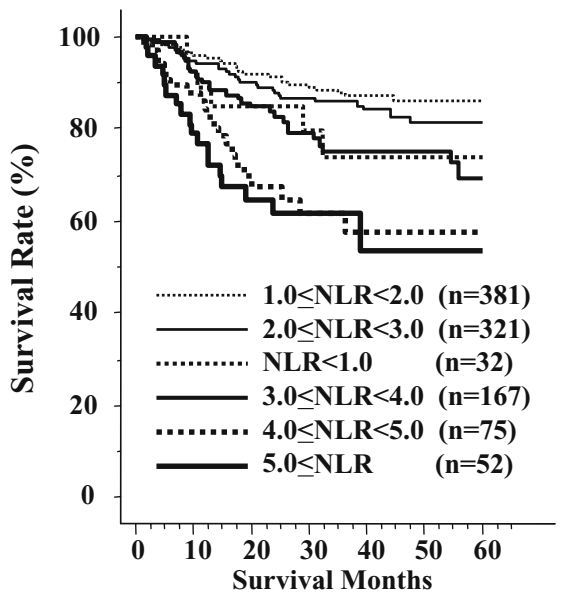

(B)

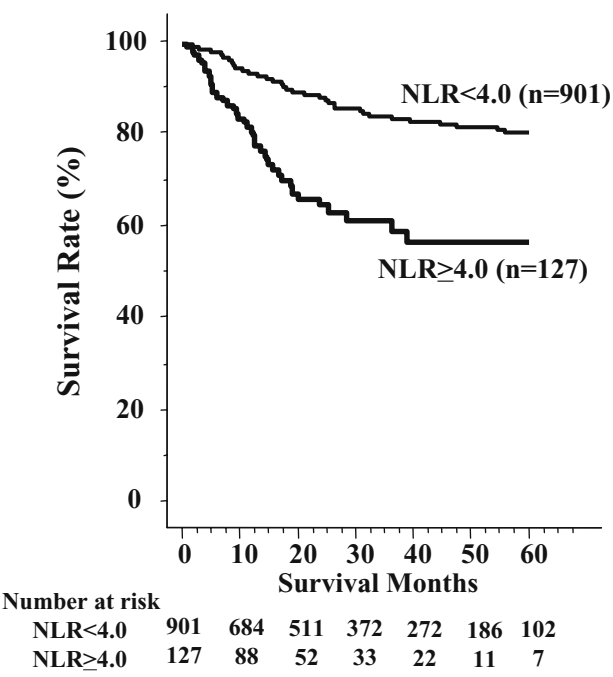

Fig. 2A,B. Kaplan-Meier survival curves, stratified by pretreatment neutrophil-lymphocyte ratio $(N L R)$, in 1028 patients with gastric carcinoma. A Divided into six groups, and $\mathbf{B}$ divided into two groups $(P<0.001)$. $P$ values were determined using the log-rank test

(A) Stage I $(p=0.035)$

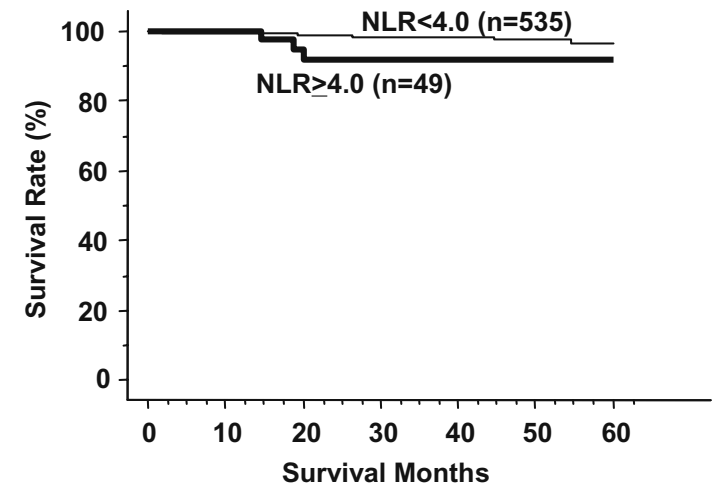

(B) Stage II $(p=0.037)$

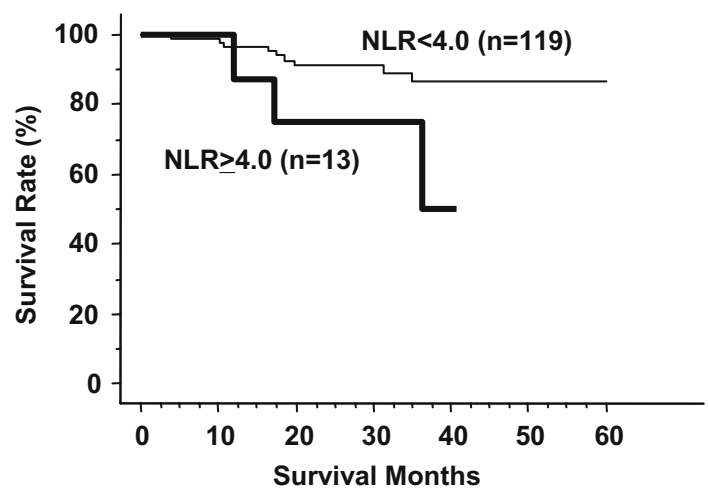

(C) Stage III $(p=0.084)$

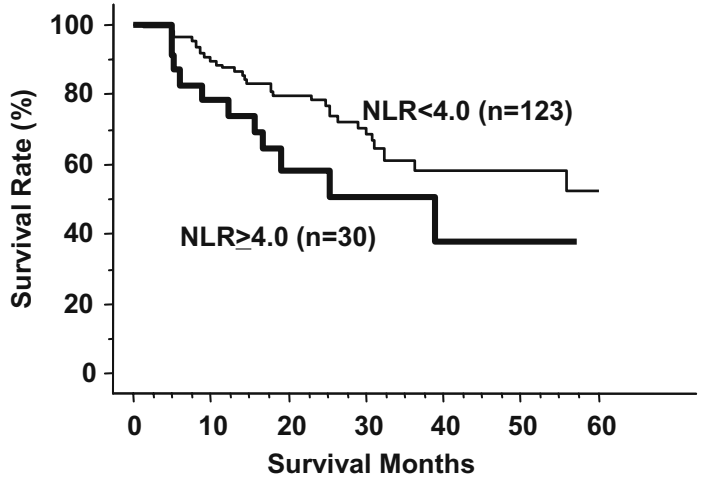

(D) Stage IV ( $p=0.005)$

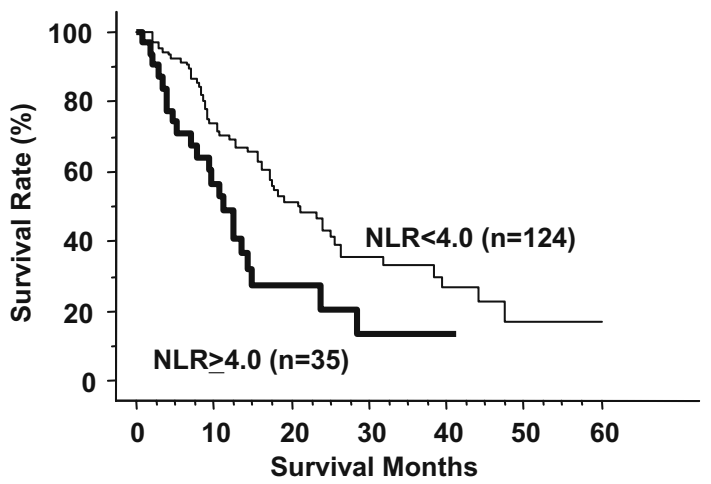

Fig. 3A-D. Kaplan-Meier survival curves of 1028 patients with gastric carcinoma, stratified by pretreatment NLR. A Stage I, $\mathbf{B}$ stage II, $\mathbf{C}$ stage III, and $\mathbf{D}$ stage IV patients. $P$ values were determined using the log-rank test 
value to distinguish patients with a poor prognosis from those with a good prognosis. Patients with NLR values between 4.0 and 5.0, revealed a survival curve similar to that of patients with the highest NLR $(5.0 \leq)$. Patients with medium NLR values (between 1.0 and 3.0) showed relatively better survival curves than those of patients with the lowest NLR values $(<1.0)$. The inconsistency of survival between the NLR $<1$ group and the $1 \leq$ NLR $<3$ group may have been due to the small number of patients in the former group. Based on univariate analysis, an NLR of 3.0 was also potentially useful as a cutoff value to predict patients' survival. When an NLR of 3.0 was used as a cutoff value in multivariate analysis, the multivariate $P$ value was 0.0309 and the adjusted hazard ratio was 1.466. On the other hand, when an NLR of 4.0 was used as a cutoff value in multivariate analysis, the multivariate $P$ value was 0.0027 and the adjusted hazard ratio was 1.845 . Although both cutoff values were statistically significant to predict patients' prognosis, an NLR of 4.0 seemed to be a more useful cutoff value than an NLR of 3.0.

A high NLR reflects an increased neutrophil count and/or a decreased lymphocyte count. Teramukai et al. [19] reported that an increased neutrophil count in itself was an independent risk factor for reduced survival in non-small cell lung cancer. In our present study of a group with gastric cancer, however, although the neutrophil count in itself was not an independent risk factor (data not shown), the NLR was selected as an independent risk factor for reduced survival.

Recent developments in cancer immunotherapy, and the relationship shown between lymphocyte-neutrophil numbers and prognosis in the present study could be related, and could be exploited to elucidate the response and effectiveness of cancer immunotherapy [20-22].

The major limitation of the present study was that the blood findings were not compared to findings such as peritumoral inflammation in the primary tumor tissue. Therefore, the notion that the increase in the NLR with stage is related to an increase in peri-tumoral inflammation is highly speculative. A comparison between the NLR and data for the numbers and specific cell types involved in peritumoral inflammation could have provided a potential functional explanation for our findings, which at present, are purely descriptive in nature. Such a comparison could have given some new insight into the relationship between the NLR and prognosis in gastric cancer patients.

The platelet count is another convenient parameter within the blood cell count that can help to predict patients' survival. An increased platelet count, thrombocytosis, was reported as an independent risk factor for reduced survival in advanced gastric cancer and esophageal cancer [6,23]. Therefore, we co-analyzed high platelet count with tumor stages and high NLR in our multivariate analysis. A high NLR was revealed to be associated with a high platelet count, and moreover, was selected as an independent risk factor for reduced survival.

Because a peripheral blood cell count is a quick and easy assay to perform, measurement of the NLR may be useful as a clinical biological marker, not only to estimate tumor load but also to predict prognosis in patients with gastric cancer.

\section{References}

1. Nakajima K, Suzuki T, Shimada H, Hayashi H, Takeda A, Ochiai T. Detection of preoperative serum anti-p53 antibodies in gastric cancer. Tumour Biol 1999;20:147-52.

2. Nakajima K, Ochiai T, Suzuki T, Shimada H, Hayashi H, Yasumoto A, et al. Impact of preoperative serum carcinoembryonic antigen, CA 19-9 and alpha fetoprotein levels in gastric cancer patients. Tumour Biol 1998;19:464-9.

3. Coussens LM, Werb Z. Inflammation and cancer. Nature 2002; 420:860-7.

4. Balkwill F, Mantovani A. Inflammation and cancer: back to Virchow? Lancet 2001;357:539-45.

5. Shimada H, Nabeya Y, Okazumi S, Matsubara H, Shiratori T, Aoki $\mathrm{T}$, et al. Elevation of preoperative serum C-reactive protein level is related to poor prognosis in esophageal squamous cell carcinoma. J Surg Oncol 2003;83:248-52.

6. Shimada H, Oohira G, Okazumi S, Matsubara H, Nabeya Y, Hayashi $\mathrm{H}$, et al. Thrombocytosis associated with poor prognosis in patients with esophageal carcinoma. J Am Coll Surg 2004; 198:737-41.

7. Ietomi K. A study on the role of granulocytes in carcinomabearing hosts $-\mathrm{G} / \mathrm{L}$ ratio as a new host indicator (in Japanese). Nippon Gan Chiryo Gakkai Shi 1990;25:662-71.

8. Satomi A, Murakami S, Ishida K, Mastuki M, Hashimoto T, Sonoda M. Significance of increased neutrophils in patients with advanced colorectal cancer. Acta Oncol 1995;34:69-73.

9. Walsh SR, Cook EJ, Goulder F, Justin TA, Keeling NJ. Neutrophillymphocyte ratio as a prognostic factor in colorectal cancer. J Surg Oncol 2005;91:181-4.

10. Sarraf KM, Belcher E, Raevsky E, Nicholson AG, Goldstraw P, Lim E. Neutrophil/lymphocyte ratio and its association with survival after completeresection in non-small cell lung cancer. J Thorac Cardiovasc Surg 2009;137:425-8.

11. Halazun KJ, Aldoori A, Malik HZ, Al-Mukhtar A, Prasad KR, Toogood GJ, et al. Elevated preoperative neutrophil-to-lymphocyte ratio predicts survival following hepatic resection for colorectal liver metastases. Eur J Surg Oncol 2008;34:55-60.

12. Gomez D, Farid S, Malik HZ, Young AL, Toogood GJ, Lodge JP, et al. Preoperative neutrophil-to-lymphocyte ratio as a prognostic predictor after curative resection for hepatocellular carcinoma. World J Surg 2008;32:1757-62.

13. Shau HY, Kim A. Suppression of lymphokine-activated killer induction by neutrophils. J Immunol 1988;141:4395-402.

14. Hirashima M, Higuchi S, Sakamoto K, Nishiyama T, Okada H. The ratio of neutrophils to lymphocytes and the phenotypes of neutrophils in patients with early gastric cancer. J Cancer Res Clin Oncol 1998;124:329-34.

15. Yamanaka T, Matsumoto S, Teramukai S, Ishiwata R, Nagai Y, Fukushima M. The baseline ratio of neutrophils to lymphocytes is associated with patient prognosis in advanced gastric cancer. Oncology 2007;73:215-20.

16. Toge T, Yamaguchi Y. Protein-bound polysaccharide increases survival in resected gastric cancer cases stratified with a preoperative granulocyte and lymphocyte count. Oncol Rep 2000;7:1157-61. 
17. Gwak MS, Choi SJ, Kim JA, Ko JS, Kim TH, Lee SM, et al. Effects of gender on white blood cell populations and neutrophillymphocyte ratio following gastrectomy in patients with stomach cancer. J Korean Med Sci 2007;22:104-8.

18. Japanese Gastric Cancer Association. Japanese classification of gastric carcinoma - 2nd English edition - Gastric Cancer 1998; 1:10-24.

19. Teramukai S, Kitano T, Kishida Y, Kawahara M, Kubota K, Komuta $\mathrm{K}$, et al. Pretreatment neutrophil count as an independent prognostic factor in advanced non-small-cell lung cancer: an analysis of Japan Multinational Trial Organization LC00-03. Eur J Cancer 2009;45:1950-8.

20. Donskov F, von der Maase H. Impact of immune parameters on long-term survival in metastatic renal cell carcinoma. J Clin Oncol 2006;24:1997-2005.
21. Donskov F, Hokland M, Marcussen N, Torp Madsen HH, von der Maase H. Monocytes and neutrophils as 'bad guys' for the outcome of interleukin-2 with and without histamine in metastatic renal cell carcinoma-results from a randomised phase II trial. $\mathrm{Br}$ J Cancer 2006;94:218-26.

22. Schmidt H, Suciu S, Punt CJ, Gore M, Kruit W, Patel P, et al. Pretreatment levels of peripheral neutrophils and leukocytes as independent predictors of overall survival in patients with American Joint Committee on Cancer stage IV melanoma: results of the EORTC 18951 Biochemotherapy Trial. J Clin Oncol 2007;25: $1562-9$.

23. Ikeda M, Furukawa H, Imamura H, Shimizu J, Ishida H, Masutani $\mathrm{S}$, et al. Poor prognosis associated with thrombocytosis in patients with gastric cancer. Ann Surg Oncol 2002;9:287-91. 